\title{
Philosophiques
}

\section{Introduction à l'histoire du concept de réflexion : position d'une recherche et matériaux bibliographiques}

\section{Robert Hébert}

Volume 2, numéro 1, avril 1975

URI : https://id.erudit.org/iderudit/203027ar

DOI : https://doi.org/10.7202/203027ar

Aller au sommaire du numéro

Éditeur(s)

Société de philosophie du Québec

ISSN

0316-2923 (imprimé)

1492-1391 (numérique)

Découvrir la revue

Citer cet article

Hébert, R. (1975). Introduction à l'histoire du concept de réflexion : position d'une recherche et matériaux bibliographiques. Philosophiques, 2(1), 131-153. https://doi.org/10.7202/203027ar d'utilisation que vous pouvez consulter en ligne. 


\title{
BULLETIN
}

\section{INTRODUCTION À L'HISTOIRE DU CONCEPT DE RÉFLEXION : \\ POSITION D'UNE RECHERCHE ET MATÉRIAUX BIBLIOGRAPHIQUES}

\author{
par Robert Hébert
}

Philosophie, histoire de la philosophie et philosophie de I'histoire de la philosophie constituent aujourd'hui une matrice privilégiée, offrant à l'analyse critique contemporaine, un vaste terrain de travail. Terrain matériel où l'image culturelle de l'homme compose un paysage habité, serein, souvent glorifié mais dont les failles singulières du non-dit, du refoulé et du conflictuel commencent à surgir dans leur irréductibilité. Quel travail ? Travail manipulateur de ce qui demeure le point commun et le lieu commun de la production philosophique, les signes; signes de croyances, signes de savoirs, signes d'illusions qui maintiennent culturellement une indéfatigable involution vers l'intelligible. Si l'exercice de la raison (d'une certaine raison) s'est diversifié de mille et une façons, utilisant la ruse des ruptures, des différenciations scolaires, des subversions sémantiques et des partis-pris culturels, il n'en a pas moins perpétué une même logique, entretenue dans les rapports continus entre l'élaboration des systèmes de pensée, la pratique institutionnelle de la philosophie et la diffusion culturelle de cet exercice de la raison. Ce qu'il faut maintenant nommer, interroger et radicaliser, c'est cette involution vers l'intelligible en tant que telle.

Réactiver pour eux-mêmes les textes et les projets originaires que les auteurs philosophiques nous ont légués ne satisfait 
plus. Un voyage lectoriel dans les archives philosophiques conserve encore son sens dans la mesure où il fait voir comment s'est constitué (et se constitue encore) le lexique culturel de la pbilosophie, lexique qui systématise dans la langue une image millénaire du zôon noèton, une volonté de savoir bien spécifique, un comportement social transmissible qui nécessairement doit prendre le détour du masque institutionnel pour se réaliser. Le voyage se fait donc là où se pratique, se communique et se définit l'exercice de la raison, c'est-à-dire là où fonctionnent l'idée, le concept et la catégorie dans le discours philosophique.

Je me suis ainsi intéressé au concept de réflexion pour des raisons stratégiques, dans la mesure où ces «raisons » incitaient à faire le travail d'une lecture méta-réflexive. 1) Dans la conversation quotidienne de l'institution philosophique, le concept de réflexion occupe une position ambiguë, ne serait-ce qu'au niveau de sa fréquence d'emploi, de son utilisation pédagogique et de son apparente neutralité : ambiguïté symptomatique qu'il faut pouvoir interpréter. 2) Le concept de réflexion participe avant tout à l'ordre de l'instauration (et non à l'ordre de la représentation); autrement dit, l'analyse critique nous amène à cerner les conditions de production-apparition du discours philosophique. 3) Il est possible de retracer l'histoire du concept de réflexion et d'y déceler une dénivellation de moments riche en perspectives théoriques; par exemple, du concept de réflexion comme signifié par le discours philosophique ( la «réflexion» dans les théories de la connaissance) au concept de réflexion comme signifiant dans le discours philosophique (les méthodes réflexives explicites). 4) En tant que valeur d'usage, le concept de réflexion inscrit le discours philosophique dans une perspective technologique, technologie dont il est nécessaire maintenant de re-penser l'origine, les articulations, la finalité et peut-être même son effet d'impouvoir. Paradigme de ce qui se constitue et se transmet dans le lexique culturel de la philosophie, le concept de réflexion est un concept clé à partir duquel une dérive radicale est possible.

Quelques remarques s'imposent sur l'organisation de ces matériaux bibliographiques. Organisation déjà signifiante, car le détour bibliographique désigne ici une manière stratégique de 
travailler le concept de réflexion dans sa densité historique. A travers les problèmes de source et de formation, mettre en cause une conception monographiste de l'histoire des idées; à travers le fait de la transmission, mettre en cause la permanence de l'institution philosophique; à travers les variations d'usage, mettre en cause le système des effets idéologiques de tout concept.

A. Cette bibliographie présente un système de renvois entre la littérature «seconde » ( au double sens de littérature d'accès et de littérature non classique) traitant spécifiquement du concept de réflexion et les textes d'auteurs classiques dispersés dans les archives philosophiques. Au lieu donc de nommer les lieux d'apparition de la « réflexion» dans ces textes, nous les avons signifiés par l'ensemble des instruments de travail qui constituent eux aussi le territoire de la pratique philosophique: lexiques, indexations d'ouvrages, articles thématiques, bibliographies d'auteurs. Par exemple, le Hegel-Lexikon de Glockner qui inscrit à l'entrée Reflexion les usages multiples de ce terme chez Hegel. Quant aux commentaires, gloses ou recherches parallèles, nous avons retenu ceux qui, par surenrichissement ou par originalité, accentuent soit une interprétation réflexive des textes classiques, soit une problématique réflexive renouvelée. A chaque ouvrage cité, nous avons pris soin de mentionner le titre et la pagination du chapitre (ou de la section) concernant le problème de la réflexion.

Les présupposés qui ont présidé à cette décision sont simples; ils déterminent d'ailleurs la position de notre recherche. 1) L'importance d'un concept en philosophie ne tient pas seulement à son utilisation dans les textes classiques mais aussi à sa résonnance dans le corpus philosophique institutionnel. 2) Il y a bien des signatures en philosophie, mais celles-ci ne fonctionnent que comme repères singuliers dans cet échange social de signes valorisés qui s'appelle le discours philosophique. Le système de renvois entre la littérature «seconde 》 et les sources « premières 》 vient en quelque sorte attester le sens de ces présupposés.

B. Nous avons orienté nos recherches vers les textes du corpus philosophique qui font du mot 'réflexion' un usage strict, c'est-à-dire que nous avons réduit au minimum l'ensemble des notions connexes et des problèmes classiques liés à l'idée de 
réflexion. Par exemple : abstraction-intuition intellectuelle, dialectique, jugement-vérité-certitude, constitution transcendantale de l'idéalisme, immédiateté-médiation, subjectivité, conscience de soi-connaissance de soi dont la littérature philosophique abonde. $\mathrm{Si}$ les textes parlant du concept de réflexion renvoient très souvent à ce vocabulaire (inclus par conséquent dans ces matériaux), l'inverse ne se vérifie pas toujours. Une telle bibliographie couvrirait alors toute l'histoire de la philosophie. A la limite, elle ne serait plus intéressante, car le bloquage par profusion viendrait neutraliser la pertinence même d'une enquête sur le concept de réflexion.

C. Le découpage de ces matériaux vise avant tout à retracer l'histoire du concept de réflexion et à dégager des problématiques bien identifiables, permettant ainsi l'analyse fonctionnelle de ses idéologies. Ajoutons également une visée pédagogique : expliciter les articulations du détour bibliographique afin qu'il se reproduise ailleurs, différemment sans doute, avec d'autres concepts du lexique philosophique.

Section I : éléments d'historiographie et de lexicographie - découpage bibliotechnique. Cette section signale quelques instruments de travail dans la littérature philosophique ou extraphilosophique (lexiques, dictionnaires, vocabulaires, encyclopédies). A partir des définitions de la réflexion données par leurs auteurs, des citations de textes classiques, des informations historiques et des maténiaux bibliographiques (très réduits dans tous les cas), il devient possible de brosser une esquisse historique du concept de réflexion. Cependant, la perspective théorique critique est généralement absente de ces instruments de travail.

Deux manques à signaler. Le premier, parce qu'il est symptomatique d'une culture philosophique différente: il s'agit de The Encyclopedia of Pbilosopby (Paul Edwards editor in chief, New York-London, Maomillan Co. \& Free Press, 1967) où le terme reflection n'a pas d'entrée. Le deuxième, parce qu'il est en voie de publication: il s'agit de la réédition enrichie et augmentée du Wörterbuch der Pbilosophischen Begriffe de Rudolf Eisler, sous le titre de Historisches Wörterbuch der Philosopbie. (hrsg. von J. Ritter, Basel-Stuttgart, Schwabe \& Co., 1971), qui comprendra neuf volumes en tout (dont trois sont déjà parus). 
Sections II-IV : connotations réflexives dans la philosophie antique et médiévale, concept de réflexion et vocabulaire philosophique du XVIIe-XVIIIe siècle - découpage selon des périodes bistoriques.

Par-delà le problème des équivalents sémantiques, la deuxième section suggère quelques analogies: le gnôti seauton, noièsis et dianoia chez Platon, koinè aisthèsis et noèsis noèseos chez Aristote, le vocabulaire de la conscience chez Plotin (synaisthèsis, parakoloutbèsis, antilèpsis), epistropbè, relation noièsis-noèton et aut'bypostaton dans le néo-platonisme, le principe d'intériorisation chez Augustin et dans la tradition du socratisme chrétien médiéval. Ces connotations réflexives sont importantes à plus d'un titre. 1) Certaines d'entre elles ont contribué à la formation de la reflexio chez Thomas d'Aquin, notion inscrite par conséquent dans la terminologie scolastique. 2) Plusieurs représentants de la tradition réflexive française ont retrouvé dans l'augustinisme le fondement historique de leur projet et de leurs discours. 3) Certains historiens de la philosophie en Allemagne (K.-H. Volkmann-Schluck, H.J. Krämer, W. Beierwaltes, K. Oehler) interprètent l'avènement de ces connotations comme la découverte de la structure réflexive de l'esprit humain.

La quatrième section regroupe les usages non systématiques du concept de réflexion dans la philosophie pré-kantienne.

Sections III-V-VI-VII : concept de réflexion et tradition thomiste, concept de réflexion et tradition de l'idéalisme allemand, concept de réflexion et tradition réflexive française, concept de réflexion et tradition phénoménologique - découpage selon des traditions philosopbiques classiques.

Mise à part la troisième section qui se rapporte au vocabulaire de la réflexion chez Thomas d'Aquin (reflexio, reditio completa, intentio secunda) et à la reprise néo-scolastique de sa problématique réflexive depuis les années 1920, les autres sections concernent trois pratiques réflexives différentes où le concept de réflexion s'identifie à des métbodes philosopbiques globales. L'élaboration d'une méthodologie comparée peut donc per. mettre d'analyser les types de fonctionnement et de finalité que le concept de réflexion s'attribue dans le discours philosophique : 
la réflexion comme élaboration systématique du Savoir, la réflexion comme expression libre de l'Agir, la réflexion comme explicitation du Vécu originaire.

Sections VIII-IX : théories générales de la réflexion, divers - découpage thématique dans la production pbilosopbique en général. La huitième section rassemble des textes qui expliciten une philosophie de la réflexion, soit avec des références culturelles à l'histoire de la philosophie, soit sans référence, comme développement d'une pensée originale. La perspective critique (par rapport à la formation et à l'usage du concept de réflexion) est généralement absente de ces textes. Bien que sa portée soit plus restreinte, la dernière section conserve la même fonction ; elle contient aussi des textes qui n'ont pu prendre place dans le schéma de notre détour bibliographique.

\section{I. ÉLÉMENTS D'HISTORIOGRAPHIE ET DE LEXICOGRAPHIE}

(a) Sources principales :

Abbagnano, N., Dizionario di filosofia, Torino, Unione Tipografico-Editrice torines,e 1964 (nouvelle édition). Voir \& riflessione 》 et « riflettente e determinante », 732-4. On trouvera d'autres éléments à «astrazione », 75-7; « autocoscienza », 87-8 et «coscienza », 178-87.

Eisler, R., Wörterbuch der Philosopbischen Begriffe, Berlin, E.S. Mittler u. Sohn, $1927-1930$ (4e édition). Voir « Reflexion », II, 657-60 et "Uberlegung 》, III, 287-90. On trouvera aussi d'autres éléments à «Apperzeption》, I, 77.84; «Selbstbewusstsein», III, 48-58 et «Warhnehmung (innere) 》, III, 483-91.

Ferrater Mora, J., Diccionario di filosofía, Buenos Aires, Ed. Sudamericana, 1965 (Se édition). Voir \& reflexión», II, 544-6.

Enciclopedia Cattolica, Città del Vaticano, Ente per l'Enciclopedia Cattolica e per il libro cattolico, 1953. Voir « riflessione» (V. Viglino), X, 902-4.

Enciclopedia filosofica, Venezia-Roma, Instituto per la collaborazione culturale, 1957. Voir «Riflessione» (A. Fossati et F. Viscidi), IV, 119-22 ; «Psicologia riflessiva» (E. Cattonaro), 111, 1741-2 et «Filosofia della riflessione 》 (A. Lantrua), 11, 424.

Lexikon für Theologie und Kirche, Freiburg im Breisgau, Verl. Herder, 1963. Voir «Reflexion》 (J.B. Lotz), VIII, 1067-9.

New Catbolic Encyclopaedia, New-York, McGraw-Hill Book Co., 1967. Voir « reflection» (R.N. Schmidt), XII, 166-8. 
(b) Sources secondaires:

Foulquié, P., Dictionnaire de la langue pbilosopbique, avec la collaboration de R. Saint-Jean, Paris, P.U.F., 1962. Voir « réflexion» et « réflexif », 620-2.

Lalande, A., Vocabulaire tecbnique et critique de la pbilosopbie, Paris, P.U.F., 1968 (10e édition). Voir à la suite « réfléchi », « réfléchissant», « réflexif » et « réflexion », 902-6.

(c) Divers :

Von Wartburg, W., Französisches Etymologisches Wörterbuch. Eine Darstellung des galloromanischen Spracbschatzes, Basel, R.G. Zbinden \& Co.. Cf. «reflectere 》 et «reflexio », X, 1962, 190-2.

The Great Books: A Syntopicon of Great Books of the Western World, M.J. Adler Ed. in Chief, W. Gorman General Ed., Chicago-London-Toronto, Encyclopaedia Britannica, 1952. Cf. \&Reflection (psychol.) 》 et « Reflexivity of mind » à l'inventaire des termes, II, 1336.

II. CONNOTATIONS RÉFLEXIVES DANS LA PHILOSOPHIE ANTIQUE ET MÉDIÉVALE.

(a) Platon et Aristote:

Oehler, K., Die Lehre vom Noetischen und Dianoetischen Denken bei Platon und Aristoteles. Ein Beitrag zur Erforschung der Geschichte des Bewusstseinsproblems in der Antike, München, Verl. C.H. Beck, 1962. Avec une im. portante bibliographie sur le sujet, 265-80; cf. «Reflexion》 à l'index, 282. Voir aussi :

Ballard, E.G., Socratic Ignorance. An Essay on Platonic Self-Knowledge, The Hague, M. Nijhoff, 1965.

Drew, A.H., «Self-reflexion and Knowing in Aristotle», Giornale di Metafisica, 23 (1968), 49-61.

Gadamer, H.-G., «Vorgestalten der Reflexion», Subjektivität und Metapbysik (= Festschrift für Wolfgang Cramer), Frankfurt am Main, V. Klostermant, $1966,128-43$.

(b) Le néo-platonisme :

Beierwaltes, W., Proklos. Grundzüge seiner Metaphysik, Frankfurt am Main, V. Klostermann, 1965. Cf. « Rückgang (Rückkehr, Reflexion, Inwendigkeit der Seele und des Geistes, Innerlichkeit. Epistrophè - noologisch und kosmologisch) 》 à l'index, 433.

Huber, G., Das Sein und das Absolute. Studien zur Gescbicbte der Ontologischen Problematik in der spätantiker Pbilosopbie, Basel, Verl. für Recht und Gesellschaft (= Studia Pbilosopbica, supp. 6), 1955. Cf. 1ère partie, sec. 1 : «Die Wandlung des Seinsbegriffs 》, 20-48.

Krämer, H.J., Der Ursprung der Geistmetaphysik. Untersuchungen zur Gescbicbte des Platonismus zwischen Platon und Plotin, Amsterdam, Verl. P. Schippers, 1964. Cf. «Reflexion a) metaphysisch ... b) philosophisch » à l'index, 470. 
Volkmann-Schluck, K.-H., Plotin als Interpret der Ontologie Platos, Frankfurt am Main, V. Klostermann, 1941. Cf. ch. V, sec. b) : « Der Weg zum 'Einung' als Rückzug des Geistes in die Reflexivität», 85-95.

Voir aussi :

Armstrong, A.H., «The background of the doctrine 'that the intelligibles are not outside the intellect' \$, Les sources de Plotin (=Entretiens sur l'Antiquité classique, V), Vandoeuvres-Genève, Fondation Hardt, 1960, 391-425.

Aubin, P., Le problème de la «conversion». Etude sur un terme commun à l'bellénisme et au christianisme des trois premiers siècles, Paris, Beauchesne, 1963. Avec une importante note bibliographique, 15-6; cf. index des epistrephein et epistrophè relevés dans l'Ancien et le Nouveau Testament, dans la tradition philosophique grecque et dans la tradition patristique, 207-31.

Baladi, N., La pensée de Plotin, Paris, P.U.F., 1970. Cf. ch. 1, sec. $1:$ : La réflexion sur l'intelligible $\gg, 19-25$.

Beierwaltes, W., « Die Metaphysik des Lichtes in der Philosophie Plotins *, Zeitschrift für Pbilosopbische Forschung, 15 (1961), 334-62.

Courcelle, P., \&Le 'Connais-toi toi-même" chez les Néo-platoniciens grecs », Le Néoplatonisme (= Colloques internationaux du C.N.R.S.), Paris, Editions du C.N.R.S., 1971, 153-66.

Hadot, P., Porphyre et Victorinus, Paris, Etudes augustiniennes, 1968. Cf. remarques sur la notion de réflexion : 1, 220, 236-8, 246, 293.

Pépin, J., «Eléments pour une histoire de la relation entre l'intelligence et l'intelligible chez Plotin et dans le néo-platonisme », Revue pbilosopbique, 146 (1956), 39-64.

Schwyzer, H.-R., « 'Bewusst' und 'Unbewusst' bei Plotin », Les sources de Plotin, op. cit., 343-78.

(c) Le socratisme chrétien :

De Bazelaire, L., "Connaissance de soi », Dictionnaire de spiritualité ascétique et mystique, Paris, Beauchesne, 1950, XII, 1511-43.

Gilson, E., L'esprit de la philosopbie médiévale, Paris, Vrin, 1969 (2e édition). Cf. ch. $\mathrm{X}$ : «La connaissance de soi-même et le socratisme chrétien », 214-33.

Ricard, R., "Notes et matériaux pour l'étude du 'socratisme chrétien' chez sainte Thérèse et les spirituels espagnols $\gg$, Bulletin bispanique, 49 (1947), 5-37, $170-204 ; 50$ (1948), 5-26; 51 (1949), 407-22.

Voir aussi :

Schöpf, A., Wabrbeit und Wissen. Die Begründung der Erkennmis bei Augustin, München, Verl. A. Pustet, 1965. Cf. 80-4, 89-91, 147-60, 163-71, 172-80, $185,192-4$.

Augustinus Magister (= Congrès international augustinien, Paris, 21-24 septembre 1954), Paris, Etudes augustiniennes, 1954, vol. I. Sur les éléments susceptibles de fournir une interprétation réflexive de l'augustinisme (l'imago Dei, la structure de la mémoire, les fonctions de l'intériorité, les trois « trini- 
tés \$ psychologiques, etc...), cf. les sections suivantes : « Philosophie augustinienne \$, 335-424 (9 articles); 《Illumination», 425.519 (8 articles) et * L'homme \$, 521-67 ( 5 articles).

(d) Divers :

Beierwaltes, W., \& Das Problem des absoluten Selbstbewusstseins bei Johannes Scotus Eriugena », Platonismus in der Pbilosopbie des Mittelalters, hrsg. von W. Beierwaltes, Darmstadt, Wissenchaftliche Buchgesellschaft, 1969, 484516.

Forest, A., « L'argument de saint Anselme dans la philosophie réflexive », Actes du Congrès international du IXe Centenaire de l'arrivée d'Anselme au Bec, Paris, Vrin, 1959, 273-94.

Malingrey, A.-M., 'Philosophia'. Etude d'un groupe de mots dans la littérature grecque des Présocratiques an IVe siècle après J.-C., Paris, C. Klincksieck, 1961.

North, H., 'Sophrosyne'. Self-Knowledge and Self-Restraint in Greek Literature, Ithaca, Cornell Univ. Press, 1966.

Schurr, A., "Vie et réflexion selon Saint Anselme », Arcbives de Pbilosopbie, 35 (1972), 111.26.

Thévenaz, $\mathrm{P}$., « $\mathrm{La}$ notion de transcendance vers l'intérieur », Annuaire de la Société suisse de philosopbie. 4 (1944), 194-217. Repris dans L'bomme et sa raison (VIf).

Volkmann-Schluck, K.-H., «Reflexion und Denken », Praxis, 5 (1969), 572-8.

\section{CONCEPT DE RÉFLEXION ET TRADITION THOMISTE}

(a) Thomas d'Aquin :

Beierwaltes, W., « Der Kommentar zum 'liber de causis' als neuplatonisches Element in der Philosophie des Thomas von Aquin 》, Pbilosopbiscbe Rundschau, 11 (1963), 192-215.

Deferrari, R.J. et al., A Lexicon of St. Thomas Aquinas Based on the 'Summa Theologica' and Selected Passages of bis Otber Works, Baltimore, J.D. Lucas Printing Co., 1948-1949. Cf. « reflecto» et « reflexio», 952-3; * intentio...5) $\gg, 586$.

Thomas d'Aquin, Somme théologique, la, qu. 84-89: La pensée bumaine, tr. par J. Webert, Paris-Tournai-Rome, Desclée de Brouwer, 1954. Cf. la note du traducteur : « La réflexion », 283-8.

Webert, J., «'Reflexio', étude sur les opérations réflexives dans la psychologie de saint Thomas d'Aquin », Mélanges Mandonnet, Paris, Vrin, 1930, I, 285. 325.

(b) Sur les travaux néo-scolastiques :

Van Riet G., L'épistémologie thomiste. Recherches sur le problème de la connaissance dans l'école thomiste contemporaine, Louvain, Bibliothèque philo- 
sophique de Louvain, 1946. On lira surtout le ch. IV, art. II : « Le recours à la réflexion de l'intelligence sur sa nature », 432-92 (avec références aux commentaires de C. Boyer, M.-D. Roland-Gosselin et B. Romeyer). Cf. «réflexion de l'intelligence sur sa nature» et « réflexion transcendantale » à l'index, 669.

Voir aussi :

De Tollenaere, M., "Méthodologie d'une cosmologie réflexive 》, Arcbives de philosopbie, 25 (1962), 229-59.

Hayen, A., La communication de l'être d'après Saint Thomas d'Aquin, Paris, Louvain, Desclée de Brouwer, 1957, 2 volumes. Sur le concept de réflexion, cf. la table méthodique des matières, sec. XII-XVI, 340-2 et sec. XXIV, 346-7.

- «La réflexion est dialogue », Congrès Bergson (= Actes du Xe Congrès des sociétés de Philosophie de langue française), Paris, Colin, 1959, 145-7.

Hoenen, P., La théorie du jugement d'après St-Thomas d'Aquin, Romae, Apud Aedes Universatis Gregorianae, 1946. Cf. ch. V : «La réflexion précédant le jugement 》, 157-91 : ch. VI : «La justification des premiers jugements », 192-216 et ch. XII : «Le 'cogito ergo sum' de St-Thomas », 330-44.

Isaye, G., "Métaphysique réflexive et philosophie de la nature», Revue internationale de philosophie, 10 (1956), 174-202.

Lonergan (B.J.), 'Verbum'. Word and Idea in Aquinas, ed. by D.B. Burrell, Notre Dame, Univ. of Notre Dame Press, 1967. Cf. ch. II : «Verbum : Reflection and Judgment $\gg, 47-95$.

- Insight. A Study of Human Understanding, New York, Philosophical Library, 1957. Cf. ch. X: «Reflective Understanding», 279-316 et 《Reflection 》 à l'index, 776.

Lotz J.B., Le jugement et l'être. Les fondements de la métaphysique, tr. par $\mathrm{R}$. Givord, Paris, Beauchesne, 1963. Cf. ch. XI, sec. 2 et surtout ch. XIII : « La réflexion parfaite en particulier, au point de vue de sa possibilité métaphysique 》, 143-61.

Marc, A., Dialectique et affirmation. Essai de métapbysique réflexive, ParisTournai, Desclée de Brouwer, 1952. Cf. «réflexive (analyse)» à l'index, 728.

- L'être et l'esprit, Paris-Louvain, Desclée de Brouwer, 1958. Cf. ch. I : "L'Idée de l'Etre: son élaboration », 17-52.

Pruche, B., «Réflexions sur la connaissance. Pour une critique existentielle d'inspiration thomiste», Revue philosopbique de Lowvain, 54 (1959), 141-83.

Régis, L.-M., Epistemology, tr. by I. Choquette Byrne, New York, Macmillan Co., 1959. Cf. « reflection 》 à l'index, 548.

(c) Sur quelques problèmes de méthodologie comparée:

De Finance, J., Cogito cartésien et réflexion tbomiste, Paris, Beauchesne, 1946.

Etcheverry, A., L'bomme dans le monde. La connaissance bumaine et sa valeur, Paris-Bruges, Desclée de Brouwer, 1963. Cf. ch. VIII : «La Méthode réflexive $\gg, 220-49$. 
Söhngen, G., Sein und Gegenstand. Das scholastische Axiom 'ens et verum convertuntur' als Fundament metaphysischer und theologischer Spekulation, Münster, Verl. der Aschendorffschen Verlagsbuchhandlung, 1930. Cf. la section intitulée: «Zur Metaphysik der Selbsterkenntnis», 279-97.

IV. CONCEPT DE RÉFLEXION ET VOCABULAIRE PHILOSOPHIQUE DU XVIIe-XVIIIe SIÈCLE.

(a) Descartes:

Sebba, G., Bibliograpbia cartesiana. A Critical Guide to the Descartes Literature 1800-1960, The Hague, M. Nijhoff, 1964. Cf. à l'index : «cogito», 445-6; «'conscience'», 447; «pensée...», 479 et «self (ego-moi) », 492. Voir aussi :

Beck, L.J., The Method of Descartes. A Study of the 'Regulae', Oxford, Clarendon Press, 1964. Cf. ch. III, sec. I : « Reflection 》, 31-6.

Gabaude, J.-M., Pbilosopbie réflexive de la volonté (=Liberté et raison. La liberté cartésienne et sa réfraction chez Spinoza et chez Leibniz, 1), Toulouse, Association des Publications de la Faculté des Lettres et Sciences humaines de Toulouse, 1970.

Krüger, G., «Die Herkunft des philosophischen Selbstbewusstseins 》, Logos, 22 (1933), 225-72.

Lewis, G., Le problème de l'inconscient et le cartésianisme, Paris, P.U.F., 1950. Cf. ch. II, sec. I : «Conscience immédiate et conscience réflexive », 107-23.

(b) Locke :

Aaron, R.I., Jobn Locke, Oxford, Clarendon Press, 1955 (2e édition). Cf. « reflection » à l'index, 319.

Cassirer, E. Das Erkenntnisproblem in der Philosopbie und Wissenschaft der neuerer Zeit, Berlin, Verl. Bruno Cassirer, 1922 (3e édition). Cf. sec. intitulée: "Sensation und Reflexion》, II, 232-53.

Klemmt, A., Jobn Locke. Theoretische Pbilosopbie, Meisenheim-Glan, Verl. A. Hain, 1967 (2e édition). Cf. ch. I, sec. 2-B : «Erfahrung, Sensation und Reflexion 》, 46-57.

(c) Spinoza :

Schuhmann, K., «Le concept de réflexion dans l'Ethique de Spinoza », Revue philosopbique de Louvain, 65 (1967), 449-66.

Voir ausssi :

Alain, Spinoza, Paris, Gallimard, 1949. Cf. sec. intitulée : «La méthode réflexive $\gg, 31-54$.

Cramer, W., Spinozas Pbilosopbie des Absoluten (= Die absolute Reflexion, I), Frankfurt am Main. V. Klostermann. 1966.

Mistahi, R., Le désir et la réflexion dans la pbilosopbie de Spinoza, Paris-LondonNew York, Gordon and Breach, 1972. 
Parkinson, G.H.R., Spinoza's Theory of Knowledge, Oxford, Clarendon Press, 1954. Cf. « reflexive knowledge » à l'index, 197.

(d) Leibniz :

Belaval, Y., «Le Problème de la Réflexion chez Leibniz », Akten des Internationalen Leibniz-Kongresses (Hannover, 14-19 November 1966), Wiesbaden, F. Steiner Verl., 1969, III, 1-19.

(e) Condillac :

Condillac, Oeuvres pbilosopbiques, éd. par G. Le Roy, Paris, P.U.F., 1947-1950. Cf. « réflexion » à l'index, III, 602.

Madinier, G., Conscience et mowvement. Etude sur la philosopbie française de Condillac à Bergson, Paris-Louvain, Béatrice-Nauwelaerts, 1967 (2e édition). Cf. ch. I : « Les orientations psychologiques et réflexives de la philosophie de Condillac », 1-38.

\section{CONCEPT DE REFLEXION ET TRADITION DE L'IDEALISME ALLEMAND.}

(a) Généralités :

Kroner, R., Von Kant bis Hegel, Tübingen, J.C.B. Mohr (Paul Siebeck), 1921 1924. Nous avons nous-mêmes indexé cet important ouvrage. Sur le concept de réflexion et ses diverses problématiques, cf. I, 42-5, 85-6, 111, 139-46, $228-36,238-56,268-76,282-4,291-8,364-74,393-4,400-1,405-6,414-6$, $424-9,435-6,460-9,479,482-3,496,502,507-13,520,524.34,541.5$, $576-7,589-92,604-6$ et II ; 13-4, 85-7, 95-6, 111-6, 138, 150-61, 163-71, $179-85,192-3,208-9,231,266,273-82,289,302-12,317-9,326-43$, $350-61,363-4,391,439,443-8,460-7,470,472-7,499,508-13$.

Schulz, W., \& Das Problem der absoluten Reflexion. Zur Auseinandersetzung mit dem deutschen Idealismus », Einsicbten (=Festschrift für G. Krüger), Frankfurt am Main, V. Klostermann, 1962, 334-60.

(b) Kant :

Eisler, R., Kant-Lexikon, Berlin, E.S. Mittler u. Sohn, 1930. Cf. \& Reflexionsbegriffe », 458-9.

Genova, A.C., « Kant's complex problem of reflective judgment 》, Review of Metapbysics, 23 (1969), 452-80.

Liedtke, M., «Der Begriff der Reflexion bei Kant», Arcbiv für Gescbicbte der Pbilosopbie, 48 (1966), 207-16.

Trotignon, P., «Les antinomies de la Raison pure et le problème de la réflexion », Revue de l'enseignement philosopbique, 14 (1964), no 4, 1-22; no 5, 1-6. Voir aussi :

De Muralt, A., La conscience transcendantale dans le criticisme kantien. Essai sur l'unité d'aperception, Paris, Aubier, 1958. 
Julia, D., «Problématique et systématique kantiennes», Revue de Métaphysique et de Morale, 64 (1959), 428-56.

Lachièze-Rey, P., L'idéalisme kantien, Paris, Vrin, 1950 (2e édition). Cf. \& téflexion 》 à l'index, 494.

Lebrun, G., Kant et la fin de la métaphysique. Essai sur la 'Critique de la faculté de juger', Paris, Colin, 1970. Cf. ch. X : «L'éclatement de la finalité technique. B) La réflexion comme méthode de la philosophie $», 263-95$.

(c) Fichte :

Baumgartner H.M. u. Jacobs, W.G., J.G. Fichte Bibliographie, Stuttgart-Bad Cannstatt, F. Frommann Verl., 1968. Cf. sec. consacrée à la Wissenschaftslebre, B 4.1, 109-15.

Fichtes Werke, hrsg. von F. Medicus, Leipzig, F. Meiner, 1912. Cf. « Reflexion», "Reflexionsform 》 et autres dérivés à l'index, VI, 667.

Horn, J.C., Monade und Begriff. Der Weg von Leibniz zu Hegel, Wien-München, Verl. R. Oldenbourg, 1965. Cf. ch. III, sec. B : « Reflektion », 106-13. Voir aussi :

Bourgeois, B., L'idéalisme de Ficbte, Paris, P.U.F., 1968.

Julia, D., «Le savoir absolu chez Fichte et le problème de la philosophie», Archives de philosopbie, 25 (1962), 345-70.

- , La question de l'bomme et le fondement de la pbilosopbie. Réflexion sur la pbilosophie pratique de Kant et la pbilosopbie spéculative de Fichte, Paris, Aubier, 1964. Sur l'utilisation du concept de réflexion, cf. 59.68, 118$41,193-230$ et $231-316$.

Reiner, P., Reflexion und Gefübl. Die Theologie Fichtes in seiner vorkantischen Zeit, Berlin, Walter de Gruyta \& Co., 1969.

Schindler, I., Reflexion und Bildung in Fichtes Wissenschaftslebre. Versucb einer Herausarbeitung systematischer Grundstrukturen der Pädagogik, Bonn, 1962.

Schuhmann, K., Die Grundlage der Wissenschaftslebre in ibrem Umrisse. Zu Fichtes 'Wissenschaftslebren' von 1794 und 1810, Den Haag, M. Nijhoff, 1968. Cf. ch. II, sec. D : «Die Reflexionsform und das Dasein », 66-70.

(d) Schelling :

F.W.J. Schellings Werke, hrsg. von Otto Weiss, Leipzig, F. Eckardt Verl., $190 \%$. Cette édition partielle des oeuvres de Schelling a le mérite de contenir un index. Cf. « Reflexion * et * Reflexionsphilosophie à l'index, III, 913.

Scbelling, System des transzendentalen Idealismus, mit eine Einleitung von W. Schulz, Hamburg, Felix Meiner Verl., 1957. Sur le problème de la réflexion, cf. introduction, IX-XLIV.

Voir aussi :

Jaspers, K., Scbelling. Grösse und Verbängnis, München, R. Piper Verl., 1955. Cf. ch. IV, sec. I : « Reflexivität \$, 222-35.

Wild, C., Reflexion und Erfabrung. Eine Interpretation der Früb- und Spätpbilosopbie Schellings, Freiburg-München, K. Alber, 1968. 
(e) Hegel :

Glockner, H., Hegel-Lexikon, Stuttgart, F. Frommanns Verl., 1957. Cf. «Reflexion 》, 11, $1991-9$.

Haering, T., Hegel, sein Wollen und sein Werk. Eine cbronologische Entwicklungsgescbichte der Gedanken und der Sprache "Hegels, Berlin-Leipzig, Teubner, 1929-1938. Cf. "Reflexion» aux index des deux tomes, I, 782-3 et II, 523.

Parmi les innombrables commentaires relatifs au problème de la réflexion, voir :

Belaval, Y., «La doctrine de l'essence chez Hegel et Leibniz», Arcbives de philosopbie, 33 (1970), 547-78.

Dubarle, D., « La Logique de la réflexion et la transition de la Logique de l'Etre à celle de l'Essence », Revue des Sciences pbilosopbiques et théologiques, 56 (1972), 193-222.

Düsing, K., «Spekulation und Reflexion. Zur Zusammenarbeit Schellings und Hegels in Jena », Hegel-Studien, 5 (1969), 95-128.

Fleischmann, E., La science universelle ou la logique de Hegel, Paris, Plon, 1968. Cf. ch. VI, sec. I : «La nature de la réflexion», 134-60.

Hartmann, N., Die Pbilosophie des deutschen Idealismus, Berlin-Leipzig, W. de Grünter, 1929. Cf. II. Hegel, ch. III, sec. 7; "Reflexion und Wesen», 230-51.

Hyppolite, J., Logique et existence. Essai sur la logique de Hegel, Paris, P.U.F., 1953. Cf. 2 e partie: "La pensée spéculative et la réflexion», 69-163.

Kopper, J., «Reflexion und Identität in der hegelschen Philosophie», KantStudien, 58 (1967), 33-53.

Marx, W., "Absolute Reflexion und Sprache », Natur und Gescbicbte (= Karl Löwith zum 70. Geburstag), Stuttgart-Berlin-Köln-Mainz, W. Kohlhammer Verl., 1967, 237.56.

Schulz, W., «Hegel und das Problem der Aufhebung der Metaphysik», Martin Heidegger zum 70. Geburstag Festschrifts, Pfullingen, Verl. G. Neske, 1959, 67.92.

(f) Divers:

Heintz, S. Über die Reflexion bei Sören Aabye Kierkegaard, Saarbrücken, Saarländische Verlagsanstalt, 1954 ('Thèse). Cf. aussi l'indexation de \& Reflexion 》 dans les Nacbscbriften, 87.

McKinnon, A., Fundamental Polyglot Konkordans tail Kierkegaards Samlede Vaerker (= Kierkegaard Indices, II), Leiden, Brill, 1971 (en danois). Cf. «Reflexion», pp. 798-804.

Voir aussi :

Apel, K.-O., «Reflexion und materielle Praxis», Hegel-Studien, Beiheft 1 (1964), 151-66.

- , "Sprache als Thema und Medium der transzendentalen Reflexion », Man and World: an International Pbilosophical Review, 3 (1970), 323-37.

Marx, W., «Zur Präsenz der transzendentalen Differenz in der dialektischen Vernunft 》, Subjektivität. und Metapbysik, op. cit. (IIa), 233-52. 
Radermacher, H., «Zum Verhältnis von Faktizität und Reflexion in der Transzendentalphilosophie », id., 253-76.

Schwarz, J., «Die cartesianische Reflexion und die Methode der Denken des deutsches Idealismus (Fichte, Schelling, Hegel) », Congrès Descartes (= Actes du IXe Congrès international de Philosophie), Paris, Hermann, 1937. Cf. III. Etudes cartésiennes, 63-9.

\section{CONCEPT DE RÉFLEXION ET TRADITION RÉFLEXIVE FRANÇAISE.}

(a) Généralités :

Lacroix, J., Panorama de la pbilosopbie franfaise contemporaine, Paris, P.U.F., 1968 (2e édition). Cf. lère partie: «Les philosophies de la réflexion», 13-96 (J. Nabert, G. Madinier, A. Ponceau, P. Ricoeur, L. Lavelle, Alain, F. Alquié, P. Lachièze - Rey, A. Marc, E. Weil, H. Duméry).

Robberechts, L., Essai sur la philosopbie réflexive, Namur-Gembloux, Secrétariat des Publications - Ed. Duculot (= Publications de la Faculté de Philosophie et Lettres de Namur), 1971. Vol. I : De Biran à Brunschvicg.

Encyclopédie française, Paris, Société nouvelle de l'Encyclopédie française, 1957. Cf. XIX, Pbilosophie et religion, lère partie, sec. A, ch. II : «Les philosophies de la réflexion ». Ce chapitre contient les articles de J. Nabert, « La philosophie réflexive», 19.04-14 à 19.06-3 et de G. Bastide, « La conversion spirituelle», 19.06-3 à 19.06-8. Voir aussi au ch. III, l'article de A. Forest, "Signification et recueillement», 19.08-10 à 19.08-14 et les éléments bibliographiques à la fin du volume, 19.B-1.

Congrès Descartes, op. cit. (Vf), VIII-IX. Analyse réflexive et transcendance (34 articles). Signalons les contributions originales de P. Decoster, «De l'analyse réflexive à l'expérience métaphysique », 32-8; C. Sganzini, « Analyse réflexive et catégories », 68-74 et J. Laird, «Reflexive and transcendent Cognition 》, 188-94.

Les Sciences et la Sagesse (= Actes du Ve Congrès des Sociétés de Philosophie de langue française), Paris, P.U.F., 1950. Cf. lère sec.: "Sagesse ct réflexion 》 ( 8 articles $), 5-36$.

(b) Sur l'utilisation de la méthode réflexive au XIXe siècle :

Brunschvicg, L., Le progrès de la conscience dans la philosopbie occidentale, Paris, P.U.F., 1953 (2e édition). Cf. II, ch. XX : "L'analyse réflexive», 555-605.

Canivez, A., Jules Lagneau, professeur de pbilosophie. Essai sur la condition du professeur de philosopbie jusqu'à la fin du XIXe siècle, Paris, Les Belles Lettres, 1965. Cf. II, ch. VII : «Le méthode réflexive de J. Lagneau», 447-96.

Fessard, G., La méthode de réflexion chez Maine de Biran, Paris, Bloud et Gay, 1938. 
Janicaud, D., Une généalogie du spiritualisme français. Aux sources de bergsonisme: Ravaisson et la métaphysique, La Haye, M. Nijhoff, 1969. Cf. « réflexion 》 à l'index, 276.

Madinier, G., Conscience et mouvement, op. cit. (IVe), Cf. ch. X : \& L'effort moteur et l'analyse réflexive chez Lachelier \$, 317-50 et ch. XI : « L'effort et la réflexion dans la philosophie de Lagneau », 351-66.

Millet, L., Le symbolisme dans la pbilosophie de Lachelier, Paris, P.U.F., 1959. Cf. ch. III, sec. I : « L'analyse réflexive de la conscience », 69-82.

Moore, F.C.T., The Psycbology of Maine de Biran, Oxford, Clarendon Press, 1970. Cf. 1ère partie, sec. 4: « Reflection», 54-76.

(c) Bergson, Blondel, Brunschvicg :

Alexander, D.W., Bergson, Pbilosopher of Reflection, London, Bowes and Bowes, 1957.

Brunschvicg, L., Le progrès de la conscience..., op. cit. (VIb), Cf. ch. XXIII, sec. 3 : «L'immanence de la réflexion $\$, 736.56$.

Duméry, H., *Blondel et la méthode réflexive », Etudes pbilosopbiques, 7 (1952), 390-5.

Husson, L., L'intellectualisme de Bergson. Genèse et développement de la notion bergsonienne d'intuition, Paris, P.U.F., 1947. Cf. « réflexion» à l'index, 235.

Jouhaud M., «E. Le Roy, le bergsonisme et la philosophie réflexive : l'Essai d'une pbilosophie première», Etudes bergsoniennes, Paris, P.U.F., 1960, V, 85-139.

Trotignon P., L'idée de vie chez Bergson et la critique de la métaphysique, Paris, P.U.F., 1968. Cf. 3e partie : \& La vie et la subjectivité intelligente de l'être réflexif \$, 285-428 et 4e partie : «Vie et savoir : la critique de la conscience réflexive et la conscience vivante $\$, 431-520$.

(d) Sur quelques systématisations du principe de réflexion (J. Paliard, J. Nabert, G. Madinier) :

Duméry, $\mathrm{H}$. , «Aspiration et réflexion 》, Etudes philosopbiques (= Jacques Paliard), 9 (1954), 420-7.

Franck, R., «Les traits fondamentaux de la méthode de Jean Nabert», Revue pbilosopbique de Louvain, 63 (1965), 97-115.

- " Deux interprétations de la méthode de Jean Nabert», id., 64 (1966), 416-35.

Madinier, G., Conscience et signification. Essai sur la réflexion, Paris, P.P.F., 1953.

- , Vers une pbilosopbie réflexive, avec une préface d'A. Forest, Neuchâtel, $\mathrm{La}$ Baconnière, 1960.

Masset, P., La dialectique de la conscience chez Jacques Paliard. Introduction à la pensée de Jacques Paliard, Paris, P.U.F., s.d. (1959). Cf. Ière partie : * Principe Général. Nature réflexive de la conscience humaine \$, 23-100. 
Nabert, J., Eléments pour une éthique, Paris, Aubier, 1962 (2e édition). Cf. livre I : \& Les données de la réflexion $\$ 19-57$ et la préface de P. Ricoeur, 5.16.

- Le désir de Dieu, Paris, Aubier, 1966. Cf. livre I : « Désir de Dieu et structures de la conscience de soi », 21-117.

Naulin, P., L'itinéraire de la conscience. Etude de la philosopbie de Jean Nabert, Paris, Aubier, 1963. Cf. lère partie, ch. II : « La méthode réflexive », 61-80.

Paliard, J., Intuition et réflexion. Esquisse d'une dialectique de la conscience, Paris, Alcan, 1925.

- , La pensée et la vie. Recherche sur la logique de la perception, Paris, P.U.F., 1951. Cf. 2e partie: "Principe \$, 55-88.

Robberechts, L., « Réflexion phénoménologique et réflexion éthique », Etudes pbilosopbiques (= Jean Nabert), 17 (1962), 403-20.

(e) Autour de la Philosophie de l'Esprit :

Bastide, G., Les grands thèmes moraux de la civilisation occidentale, Paris, Bordas, 1958. Cf. ch. I, sec 3: «La sagesse réflexive», 16-21.

- Traité de l'action morale, Paris, P.U.F., 1961. 1. Analytique de l'action morale. Cf. livre I, ch. IV : «La provocation réflexive \$ 87-107. On lira aussi le compte-tendu de $\mathrm{A}$. Voelke, "Action morale et analyse réflexive selon G. Bastide », Studia pbilosopbica, 23 (1963), 212-29.

- , "Naturalisme et spiritualité. Le statut de la réflexion dans la pensée de Teilhard de Chardin 》, Etudes pbilosopbiques, 20 (1965), 409-47.

Forest, A., Consentement et création, Paris, Aubier, 1943. Parmi les études annexes, cf. sec. I : « Nature et rôle de la réflexion 》 $95-116$ et sec. II : *L'attention et la philosophie réflexive 》, 117-25.

Lachièze-Rey, P., Le moi, le monde et Dieu, Paris, Aubier, 1950 (nouvelle édition). Cf. essai III : * Réflexion sur la portée ontologique de la méthode de régression analytique $\gg, 183-91$.

Lavelle, L., De l'acte, Paris, Aubier, 1937. Cf. 1ère partie : \& La méthode \$ 9-57.

- "Acte réflexif et acte créateur », Bulletin de la Société française de philosopbie 36 (1936), no 4. Avec discussion.

- Manuel de métbodologie dialectique, Paris, P.U.F., 1962. Cf. livre I : \& La réflexion 》, 3.55.

Le Senne, R., Obstacle et valeur, Paris, 1934. Cf. ch. III : « Le passage de la spontanéité à la réflexion », 117-60.

Nédoncelle, M., Conscience et Logos, Paris, Editions de l'Epi, 1961. Cf. sec. intitulée: «Les équivoques de la réflexion philosophique », 157-76.

(f) Philosophies réflexives et méthodologie comparée: (P. Ricoeur, P. Théve* naz) :

Ricoeur, P., Histoire et vérité, Paris, Seuil, 1955. Cf. 2e partie, sec. IV : « Puissance de l'affirmation $\gg, 289-332$. 
- Finitude et culpabilité, Paris. Aubier, 1960, I. L'bomme faillible. Cf. ch. II : «La synthèse transcendantale 》, 35-63.

- , De l'interprétation. Essai sur Freud, Paris, Seuil, 1965. Cf. livre I, ch. III : "Méthode herméneutique et philosophie réflexive», 45-63.

- , Le conflit des interprétations. Essais d'berméneutique, Paris, Seuil, 1969. Cf. en particulier les deux sec. intitulées : « Herméneutique des symboles et réflexion philosophique 》 (1-2), 283-329.

Thévenaz, P., L'bomme et sa raison, Neuchâtel, La Baconnière, 1956. I. Raison et conscience de soi. Cf. sec. 1 : «Réflexion et conscience de soi », 29-121. Voir aussi la préface de P. Ricoeur, 9-26.

- , «'expérience réflexive de la liberté», La liberté (= Actes du IVe Congrès des Sociétés de philosophie de langue française), Neuchâtel, La Baconnière, 1949, 52-7.

Tilliette, X., «Réflexion et symboles. L'entreprise philosophique de Paul Ricoeur $\gg$, Archives de pbilosopbie, 24 (1961), 574-88.

(g) Sur l'utilisation particulière du concept de réflexion dans les \& introductions à la philosophie » et dans la tradition scolaire :

Flam, L., Passé et avenir de la philosopbie, Bruxelles, Editions de l'Institut de Sociologie (U.L.B.), 1970. Cf. ch. XI : «La réflexion philosophique», 197-219.

Sauvage, M., L'aventure pbilosopbique, Paris, Buchet-Chastel, 1966, Cf. sec. 1 : «Réflexions sur la réflexion», 123-38.

Vialatoux, J., L'intention philosopbique, Patis, P.U.F., 1969 (8e édition). Cf. ch. III, sec. 2 : «La méthode philosophique: méthode réflexive», 59-93. Voir aussi :

Balmès, R., Leçons de philosopbie, Paris, Editions de l'Ecole, 1965. Cf. I, sec. 1 de l'introduction: « $\mathrm{La}$ réflexion», 26-32.

Foulquié, P., La connaissance, Paris, Editions de l'Ecole, 1962 (nouvelle édition). Cf. sec. intitulée: « $\mathrm{La}$ philosophie comme réflexion», 43-8.

Meynard, C., La connaissance (Terminales A), Paris, Librairie classique E. Belin, 1963. Cf. sec. intitulée : « La réflexion philosophique », 23-4.

(h) Divers :

Blanché, R., Les attitudes idéalistes, Paris, P.U.F., 1949. Cf. ch. II : « Réflexion », 26-67.

Chabal, R., Vers une antbropologie pbilosopbique, Paris, P.U.F., 1964, I. Conscience et savoir. Cf. sec. intitulée : «Quel est le pouvoir de la réflexion?», $15-33$.

Duméry, H., Critique et religion. Problèmes de méthode en philosophie de la religion, Paris, Sedes, 1957. Cf. ch. VI : «L'analyse réflexive et critique (méthode de discrimination) 》, 161-77.

- , Philosophie de la religion. Essai sur la signification du cbristianisme, Paris, P.U.F., 1957. Cf. I, lère partie, ch. II : «Le Moi spirituel », 35-49. 
Lacroix, J., Le sens du dialogue, Neuchâtel, La Baconnière, 1965 (4e édition). Cf. sec. intitulée : «La réflexion》, 128-33.

Laporte, J., La conscience de la liberté, Paris, Flammarion, 1947. Cf. livre III, ch. III : «La réflexion », 161-81.

Lechat J., Analyse et synthèse, Paris, P.U.F., 1962. Cf. ch. VII : "L'analyse réflexive 》, 85-98.

Piguet, J.-C., «Analyse réflexive et langage», Studia philosopbica, 19 (1959), 193-213.

VII. CONCEPT DE RÉFLEXION ET TRADITION PHÉNOMÉNOLOGIQUE.

(a) Généralités :

Spiegelberg, H., The Pbenomenological Movement. A Historical Introduction, The Hague, M. Nijhoff, 1965. (2e édition). Cf. « reflection» à l'index, II, 724-5.

(b) Husserl :

Brand, G., Welt, Icb und Zeit. Nach unveröffentlichten Manuskripten Edmund Husserls, Den Haag, M. Nijhoff, 1955. Sur la relation entre réflexion et temporalité, cf. sec. 12-14, 61-75.

Broekman, J.M., Pbänomenologie und Egologie. Faktisches und transzendentales Ego bei Edmund Husserl, Den Haag, M. Nijhoff, 1963. Cf. ch. IV: «Edmund Husserls Subjektivitätanalyse; egologische Strukturen», 109-37.

Farber, M., « Modes of Reflection. Inscribed to the Memory of Edmund Husserl », Pbilosopby and Pbenomenological Research, 8 (1947-48), 588-600.

Hoeres, W., «Zur Dialektik der Reflexion bei Husserl», Salzburger Jabrbuch für Pbilosopbie und Psychologie, 2 (1958), 211-30.

Lévinas, E., La théorie de l'intuition dans la pbilosopbie de Husserl, Paris, Vrin, 1963 (nouvelle édition). Cf. ch. VII : «L'intuition philosophique »175-215.

Murphy, R.T., «Husserl and Pre-reflexive constitution», Pbilosopby and Pbenomenological Research, 26 (1965-66), 100-5.

Vuillemin, J., «Le problème phénoménologique: intentionalité et réflexion», Etudes philosophiques, 14 (1959), 462-70.

Voir aussi :

Asemissen, H.U., «Egologische Reflexion 》, Kant-Studien, 50 (1958-58), 262-72.

Berger, G., Le Cogito dans la pbilosopbie de Husserl, Paris, Aubier, 1941.

Bernarski, J., «Deux aspects de la réduction husserlienne : abstention et retour», Revue de Métaphysique et de Morale, 64 (1959), 337-55. Cf. en particulier la 2e partie: "La réduction comme réflexion », 346-55.

Farber, M., The Foundation of Phenomenology. Edmund Husserl and the Quest for a Rigorous Science of Pbilosopby, Cambridge, Harvard Univ. Press, 1943. Cf. «reflection» et «reflexive predicament» à l'index, 584. 
- Pbenomenology and Existence. Toward a Pbilosopby witbin Nature, New York-Evanston-London, Harper Torchbooks, 1967. Cf. ch. I : \& The Role of Reflection in Phenomenology $\$ 1$ 1-13.

Fink, E., Studien zur Pbänomenologie 1930-1939, Den Haag, M. Nijhoff, 1966. CF. 121-3, 218-23.

Funke, G., \& Bewusstseinswissenschaft. Evidenz und Reflexion als implikate der Verifikation », Kant-Studien, 61 (1970), 433-66.

Sokolowski, R., The Formation of Husserl's Concept of Constitution, The Hague, M. Nijhoff, 1964. Cf. « reflection à l'index, 248.

(c) Heidegger, Jaspers, Scheler :

Heidegger, M., Qúappelle-t-on penser ?, tr. par A. Becker et G. Granel, Paris, P.U.F., 1959. Cf. 34-8, 97.

Jaspers K., Allgemeine Psychopathologie, Berlin-Göttingen-Heidelberg, Springer Verl., 1953 (Ge édition). Cf. livre II, ch. I, sec. 4: «Selbstreflexion》, 289-96. Voir aussi «Reflexion》 et « Reflexivität» à l'index, 741.

- , Psychologie der Weltanschaumnen, Berlin, Springer Verl., 1922. Cf. ch. I, sec. 2 : «Selbstreflektierte Einstellungen», 90-117.

— , Pbilosopbie, Berlin-Göttingen-Heidelberg, Springer Verl., 1948 (2e édition). Cf. « Reflexion » à l'index, 902.

- Von der Wabrbeit, München, R. Piper Co. Verl., 1947. Cf. « Reflexion» et «Selbstreflexion》 à l'index, 1088.

Scheler, M., Le formalisme en étbique et l'étbique matériale des valeurs. Essai nouveau pour fonder un personnalisme étbique, tr. par M. de Gandillac, Paris, Gallimard, 1955 (7e édition). Cf. «Réflexion (ou Connaissance réflexive) 》, à l'index, 629 .

(d) Sur le concept de réflexion et la phénoménologie existentielle pratiquée en France :

Bannan, J.F., \& Philosophical Reflection and the Phenemenology of MerleauPonty \$, Review of Metapbysics, 8 (1954), 418-42.

Caillois, R., «Note sur l'analyse réflexive et la réflexion phénoménologique. A propos de la 'Phénoménologie de la perception' de Maurice Merleau-Ponty », Deucalion, no 1 (1946), 127-39.

De Greef, J., "Ethique, réflexion et histoire chez Lévinas », Revue pbilosopbique de Louvain, 67 (1969), 431-60.

Dufrenne, M., Phénoménologie de l'expérience esthétique, Paris, P.U.F., 1966 (2e édition). II. La perception esthétique. Cf. 3e partie, ch. III : «Réflexion et sentiment dans la perception en général » et ch. IV : « Le sentiment et la profondeur de l'objet esthétique », 462-526.

Gusdorf, G., Mytbe et métapbysique. Introduction a la pbilosopbie, Paris, Flammarion, 1953. 
Kwant, R.C., From Pbenomenology to Metaphysics. An Inquiry into the Last Period of Merleau-Ponty's Pbilosopbical Life, Pittsburgh, Duquesne Univ. Press, 1966. Cf. ch. III, sec. 2 : «Merleau-Ponty's Criticism of Reflection 》, 114-29.

Pax, C.V., «Philosophical Reflection: Gabriel Marcel» New Scboolman, 38 (1964) 159-77.

Thévenaz, P., De Husserl à Merleau-Ponty. Qu'est-ce que la phénoménologie?, Neuchâtel, La Baconnière, 1966. Cf. ch. IV, sec. 1 : «La 'réflexion radicale' ou la 'phénoménologie de la phénoménologie' $\$, 103-10$.

Troisfontaines, R., De l'existence à l'être. La philosopbie de Gabriel Marcel, Paris-Louvain, Béatrice-Nauwelaerts, 1968. Cf. t. I, ch. VII, sec. 2 : \& Réflexion \$, 199-210.

Varet , G., L'ontologie de Sartre, Paris, P.U.F., 1948. Cf. 2e partie: « La dialectique réflexive », 75-179.

VIII. THÉORIES GÉNÉRALES DE LA RÉFLEXION.

D'Arcy P., La réflexion, Paris, P.U.F., 1972.

Grue-Sorensen, K., Studier over refleksivitet. En filosofisk afbandling, Kobenhavn, J.H. Schultz Forlag, 1950 (en danois). Cf. « refleksivitet (refleksiv) 》 à l'index, 238.

Günther, G., « Metaphysik, Logik und die Theorie der Reflexion », Archiv für Pbilosopbie, 7 (1957), 1-44.

- , \& Die aristotelische Logik des Seins und die nicht-aristotelische Logik der Reflexion 》, Zeitschrift für Philosopbische Forscbung, 12 (1958), 360-407.

Hodgson, S.H., The Pbilosopby of Reflection, London, Longmans Green Co., 1878, 2 volumes.

Hoeres, W., Sein und Reflexion, Würzburg, K. Triltsch, 1956.

Misrahi, R., Lumière, Commencement, Liberté, Paris, Plon, 1969. Cf. la recension de M.B. Madaule : « Lumière, Commencement, Liberté », Revue de Métaphysique et de Morale, 75 (1970), 96-105.

Robef, E., De l'analyse réflexive, Paris, Jouve, 1925.

Varet, G., La Réflexion: Esquisse d'une problématique, Paris, Université de Paris, 1956 (Thèse).

Wagner, H., Philosopbie und Reflexion, München-Basel, E. Reinhardt Verl., 1959. Cf. les recensions de W. Cramer: \& Hans Wagner, Philosophie und Reflexion \$, Philosopbische Rundscbau, 11 (1963), 68-90 et G.A. Schrader : « Philosophy and Reflection: Beyond Phenomenology», Review of Metapbysics, 15 (1961), 81-107; voir aussi celles de W. Brugger dans Scholastik, 35 (1960), 562-6 et de H. Kunz dans Studia philosopbica, 21 (1961), 266-71. 


\section{DIVERS.}

Ambacher, M., Métbode de la philosopbie de la nature, Paris, P.U.F., 1961. Cf. ch. IV : «De l'intelligibilité abstraite à l'intelligibilité réflexive» 119-40. Voir aussi ch. V, 141-70.

Branshard, B., The Nature of Thought, London, Allen and Unwin, 1955. Cf. II, livre III ; «The movement of reflection», en particulier ch. XIX, 37-63.

Cassirer, E., The Pbilosopby of Symbolic Forms, tr. by R. Manheim, New Haven-London, Yale Univ. Press, 1953-1957. Vol. I : Language, 147-55; vol. II : The Phenomenology of Knowledge, cf. «Reflection 》 à l'index, 496.

Dempf, A., Theoretische Antbropologie, Bern, A. Francke Ag. Verl., 1950. Cf. ch. VI : «Selsthewusstseinstheorie », 165-91.

Dewey, J., The Quest for Certainty: A Study of the Relation of Knowledge and Action, New York, Minton, Balch \& Co., 1929. Cf. «Reflective Thinking » à l'index, 317.

- , How We Tbink. A Restatement of the Relation of Reflective Thinking to the Educative Process, Chicago, Gateway Ed., 1971 (nouvelle édition).

Gadamer, H.-G., Wabrbeit und Methode. Grundzüge einer pbilosopbischen Hermeneutik, Tübingen, J.C.B. Mohr (Paul Siebeck), 1965 (2e édition). Cf. «Reflexion》 et «Reflexionsphilosophie» à l'index, 521.

Giorgi, R., La riflessione simbologica, Roma, Leria Edidore, 1968.

Guzzo, A., L'io e la ragione, Brescia, Morcelliana, 1947. Cf. 3e partie, ch. V: «La riflessione 》, 152-66. Voir aussi «Riflessione 》 et "Riflettere 》 à l'index, 377.

Mead, G.H., The Pbilosopby of the Act, ed. with an Introduction by C.W. Morris and others, Chicago, Univ. of Chicago Press, 1938. Cf. la sec. intitulée : «Fragments on the Process of Reflection», 79-91. Voir aussi «Reflection» et «Reflective experience » à l'index, 689.

- , Mind, Self and Society from the Standpoint of a Social Bebaviorist, ed. with an Introduction by C.W. Morris and others, Chicago, Univ. of Chicago Press, 1934. Cf. sec. 13: «The Nature of Reflective Intelligence», 90-100 et l'essai supplémentaire III : «The Self and the Process of Reflection», 354-78.

Schulz, W., Wittgenstein. Die Negation der Pbilosopbie, Pfullingen, G. Neske, 1967. Cf. sec. intitulée: \&Die Destruktion des denkend-reflektierenden Subjekts 》, 29-39.

Teilhard de Chardin, P., Le pbénomène bumain (= Oewvres, 1), Paris, Seuil, 1955. Cf. 3e partie, ch. I, sec. 1 : "Le Pas de la Réflexion», 180-203.

- L'activation de l'énergie ( $=$ Oeuvres, 7), Paris, Seuil, 1963. Cf. sec. intitulée : «La Réflexion de l'Energie », 335-53. Voir aussi, 397-406, 419-29.

Trotignon, P., Réflexion et immédiat. Introduction à une théorie générale de la subjectivité, Paris, Université de Paris, 1969 (Thèse).

Von Hartmann, E., Kategorienlebre, Leipzig, F. Meiner, 1923 (2e édition). 
II. Die Kategorien des Reflektierenden Denkens.

Voir aussi :

Child, A., «Reflection: Its Nature and its Philosophic Import», Pbilosopby and Pbenomenological Research, 19 (1958-1959), 1-15.

Dray, W., «R.G. Collingwood on reflective Thought», Journal of Philosophy, 57 (1960), 157-63.

Grant, N.J., «Ryle and Reflection», Journal of the British Society for Pbenomenology, 1 (1970), 24-9.

Henrich, D., «Selbstbewusstsein. Kritische Einleitung in eine Theorie », Hermeneutik und Dialektik, op. cit. (Ve). I, 257-84. Cf. pour l'ensemble du Festschrift, «Reflexion 》, «Reflexionsphilosophie 》 et « Reflexionstheorie 》 à l'index, II, 377.

Montréal, décembre 1973 